\title{
Tafonomía del gasterópodo cf. Donaldina robusta (Heterobranchia: Streptacididae) del Pensilvánico Medio, Formación La Joya, Sonora, México
}

\author{
Catalina Gómez E. ${ }^{1}$, Blanca Buitrón S. ${ }^{1}$ \& Daniel Vachard ${ }^{2}$
}

1. Departamento de Paleontología, Instituto de Geología, Universidad Nacional Autónoma de México, 04510 México D. F. Fax 52 (55)55508432; c_gomez@fciencias.unam.mx, blancab@servidor.unam.mx

2. Unité de Formation et Recherche Sciences de la Terre, Université de Lille 1, 59655 Villeneuve d'Ascq Cedex, France. F; daniel.vachard@univ-lille1.fr

Recibido 05-II-2009. Corregido 07-VII-2009. Aceptado 07-VIII-2009.

\begin{abstract}
Taphonomy of the gastropod cf. Donaldina robusta (Heterobranchia: Streptacididae) from the Middle Pennsylvanian, La Joya Formation, Sonora, Mexico. Gastropods are an important component in most of the fossil record; however, investigations have focused mainly on the characterization of the tafofacies and signatures in determined environments. We present qualitative and quantitative taphonomic data for the gastropod cf. Donaldina robusta assemblages from the La Joya Formation of the Sierra Agua Verde, Sonora State, (NW) Mexico. We analyzed 176 shells. Good preservation received a high taphonomic grade (A) and poor preservation a D. The shells were complete in $72 \%$ of cases (taphonomic grade B). Less than $10 \%$ are corroded or are parallel to the layer (grade A). This rock is petrographycally classified as wackestone, sedimentologically it is characterized by middle sorting (grade B) and low grading (grade A). The fossiliferous assemblage grades as A and B. Biostratinomic features of the skeletal assemblage are characteristic of sedimentologic concentrations of autochthonous-parautochthonous elements at the accumulation site. There was minimal reworking and transport in an environment of low energy, locally produced during a short period of accumulation. Rev. Biol. Trop. 58 (1): 183-194. Epub 2010 March 01.
\end{abstract}

Key words: biostratinomy, taphonomic grades, paleoecology, gastropods, Pennsylvanian, Mexico.

Los gasterópodos son un componente muy importante en el registro fósil, a lo largo de todo el tiempo geológico, por lo cual han sido utilizados para realizar diversos estudios tafonómicos que destacan la necesidad de entender los factores que afectan su representatividad (Walker \& Carlton 1995, Zuschin et al. 2003). Las investigaciones se han centrado principalmente en la caracterización de las tafofacies y firmas tafonómicas en ambientes particulares, en la conducta hidrodinámica de las conchas y en la cuantificación de la fidelidad en los depósitos a través de estudios actualísticos.

La tafonomía abarca dos aspectos, la biostratinomía y la fosildiagénesis. La biostratinomía estudia la historia sedimentaria de los restos orgánicos y la fosildiagénesis se encarga de los procesos diagenéticos tempranos y tardíos de los restos (Speyer \& Brett 1988).

En los últimos años, los tafónomos han incrementado sus estudios para mejorar la calidad de la interpretación paleoambiental al considerar los estados de preservación de los restos orgánicos, los probables patrones de daño y el tipo de concentraciones esqueletales (Kidwell et al. 2001), orientados hacia los análogos modernos como medio de identificación y cuantificación.

Los temas que recurrentemente se estudian en esta disciplina son la identificación de procesos específicos que dejan marca en los restos orgánicos (firmas tafonómicas y tafofacies), las circunstancias que preservan a algunas especies y a otras no en un conjunto fósil 
(representatividad y fidelidad), el transporte de los restos orgánicos y la cantidad de tiempo representado en un único conjunto fósil (promedio temporal) (Behrensmeyer 1984).

El Pensilvánico está caracterizado por presentar frecuentes fluctuaciones en el nivel del mar que originaron secuencias sedimentarias regulares y cíclicas denominadas ciclotemas. Los ciclotemas han sido objeto de detallados estudios estratigráficos y sedimentológicos y se encuentran bien caracterizados e identificados (Heckel 1983, 1986, Carlson 1994).

La presente investigación tiene como objetivo el estudio tafonómico de los moluscos gasterópodos de la especie cf. Donaldina robusta (Stevens 1858) con el propósito de reconocer los procesos biostratinómicos que dieron origen a esta acumulación.

\section{MATERIALES Y MÉTODOS}

Área de estudio: En el noreste de Sonora en la Sierra Agua Verde, se encuentra expuesta una secuencia pensilvánica con características de un sistema transgresivo (TST) depositado en una plataforma subsidente (Almazán et al. 2007). La Sierra Agua Verde está situada en la Provincia de la Sierra Madre Occidental dentro de la Subprovincia Sierras y Valles del Norte, a $110 \mathrm{~km}$ al este de Hermosillo. Está constituida por rocas paleozoicas de plataforma, las rocas pensilvánicas forman parte de la Formación La Joya con una potencia de más de 294m (Ochoa \& Sosa 1993). La litología consiste en caliza fosilífera, lutita calcárea y lodolita con intercalaciones de cuerpos irregulares de pedernal.

El afloramiento estudiado se localiza a los $109^{\circ} 51^{\prime} 00^{\prime \prime} \mathrm{N}$ y $29^{\circ} 14^{\prime} 00^{\prime \prime} \mathrm{W}$. Estas rocas contienen una gran cantidad de fósiles que incluyen algas filoides, fusulínidos, esponjas hipercalcificadas (chaetétidos), corales solitarios y coloniales, braquiópodos, gasterópodos, crinoides y conodontos (Almazán et al. 2007, Buitrón et al. 2007). El análisis bioestratigráfico sugiere una edad del Pensilvánico Medio (Moscoviano) por la presencia de los fusulínidos característicos de la biozona A3 de Wilde (1990) entre los que destacan Fusulinella llanoensis, Fusulinella thompsoni y Nipperella sp. (Gómez et al. 2008).

Métodología: Se realizaron cuatro salidas al campo, en octubre de 2003, marzo de 2004, abril de 2005 y octubre de 2006, se levantó una sección estratigráfica de $100 \mathrm{~m}$ en el área de la Formación La Joya que aflora en el Rancho Agua Caliente. En ésta se ubicó a los gasterópodos a $83 \mathrm{~m}$ de la base en un estrato de caliza masiva de $1.2 \mathrm{~m}$ (Fig. 1, 2).

Los datos que se tomaron en campo fueron: el tipo de fosilización y el grado de preservación de los gasterópodos, el tipo de roca, la posición dentro del estrato y el ángulo de orientación de los ejemplares. También se midió el alto y ancho de los gasterópodos completos para determinar la distribución de tamaños e inferir si las conchas fueron producto de una concentración hidrodinámica o un efecto poblacional.

El ángulo de orientación de los organismos se midió utilizando como referencia la base del estrato, debido a que estos datos fueron tomados en sección cruzada y porque los organismos presentan un esqueleto con ejes elongados lo cual permite diferenciar claramente la posición paralela, perpendicular u oblicua de los restos (Kidwell et al. 1986). Se tomaron muestras para identificar taxonómicamente a los gasterópodos y para el estudio de láminas delgadas con el objetivo de identificar el tipo de caliza de acuerdo a la clasificación propuesta por Dunham (1962).

La descripción de las características biostratinómicas se hizo por medio de la aplicación de los grados tafonómicos, debido a que éstos resultan muy útiles para determinar la historia deposicional de estratos individuales, con un grado de resolución por debajo del de las tafofacies, que generalmente involucran escalas en orden de metros (Brandt 1989).

Los grados tafonómicos se definen utilizando cuatro características tafonómicas de los organismos, que son: grado de fractura, articulación (este criterio no aplica en gasterópodos), corrasión y orientación; y tres características sedimentológicas que incluyen el porcentaje de matriz, clasificación y gradación de la roca. 


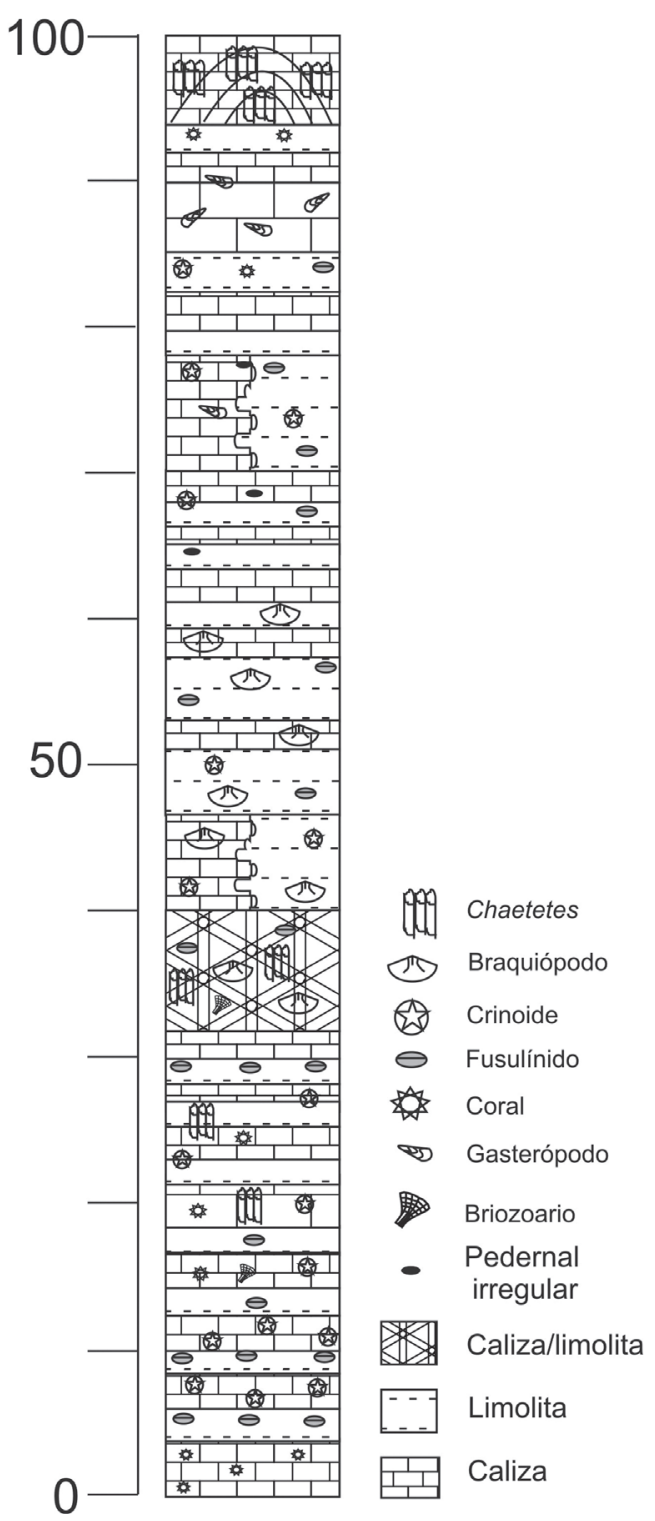

Fig. 1. Columna estratigráfica de la Formación La Joya, Sierra Agua Verde, Sonora, México.

Fig. 1. Stratigraphic column of La Joya Formation, Sierra Agua Verde, Sonora, Mexico.

El grado de fractura se refiere a la proporción de ejemplares intactos versus ejemplares rotos, la articulación solo aplica a organismos que presentan esqueletos multisegmentados. La

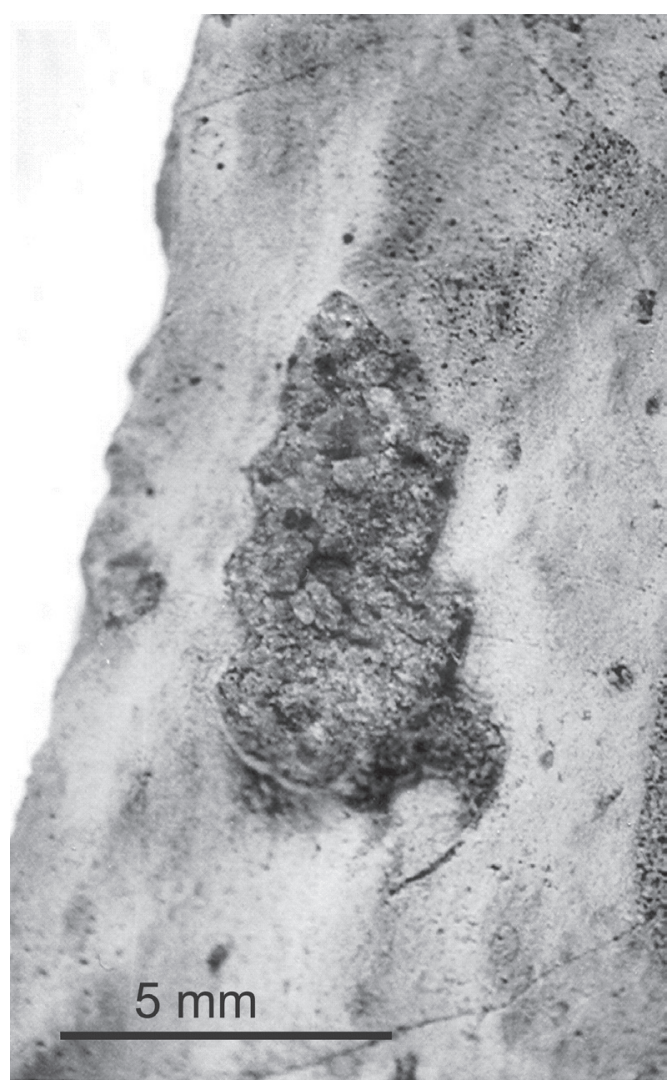

Fig. 2. Gasterópodo silicificado cf. Donaldina robusta.

Fig. 2. Silicified gastropod cf. Donaldina robusta.

corrasión es el grado de degradación general de la superficie de un resto cuando no se pueden separar los posibles causantes de disolución, bioerosión (corrosión) y abrasión, el índice de corrasión es una combinación de abrasión mecánica, corrosión química y biológica (Brett \& Baird 1986). La orientación es la medida de alineación paralela en fósiles de forma elongada.

En relación a las características tafonómicas se establecieron cuatro grados tafonómicos A, B, C y D, en las características sedimentológicas el grado $\mathrm{D}$ se subdivide en $\mathrm{D}_{1} \mathrm{y}_{2}$. $\mathrm{El}$ grado tafonómico mas alto es el $\mathrm{A}$, y caracteriza a los estratos mejor preservados y con menor índice de retrabajo, el grado tafonómico más bajo es el D y caracteriza a los conjuntos 
intensamente transportados y con pobre preservación (Brandt 1989).

La conjunción de estas características permite identificar la calidad de preservación de los fósiles y también inferir la escala relativa del tiempo aproximado de acumulación.

\section{RESULTADOS}

Se encontró un total de 235 ejemplares, de los cuales $47 \%$ se hallaban completos, considerando como completos a los organismos que presentaban al menos el $90 \%$ de su forma original (Davies et al. 1990), 28\% incompletos, y el $25 \%$ restante se consideró como indefinido al observarse únicamente en corte transversal. Los ejemplares recolectados para la identificación taxonómica fueron depositados en la Colección de Paleontología, del Departamento de Geología de la Universidad de Sonora, con los números de catálogo USON101-125.

Para el análisis tafonómico se descartaron 56 ejemplares indefinidos y se estudiaron únicamente 176 ejemplares que se encontraban completos e incompletos. De este mismo número de ejemplares se realizó un histograma y curva de frecuencia que indica la fluctuación de la anchura de la concha (Fig. 3).

De acuerdo a la clasificación de Dunham (1962) la caliza corresponde a un "wackestone" con una matriz homogénea, soportada por lodo, con una dolomitización secundaria de tipo sucroide, con bioclastos de gasterópodos, escasos braquiópodos y con abundantes microfracturas carbonatadas.
Preservación del material: La concentración de gasterópodos está caracterizada por conchas bien conservadas, prístinas, sustituidas completamente por sílice diagenético, soportadas por matriz calcárea y que retienen elementos de la ornamentación. En ningún organismo se presentó evidencia macroscópica de bioerosión o incrustación. La fragmentación en el $28 \%$ de los ejemplares se observó únicamente en el punto más débil de la estructura que corresponde a la espira (Fig. 4). La abrasión física es inconspicua.

Las conchas tienen una orientación aleatoria (Fig. 5, Apéndice 1), encontrándose menos del $10 \%$ en posición paralela al estrato.

La fábrica es dispersa y los restos se encuentran soportados por matriz. Presenta una pobre clasificación esqueletal, pues los tamaños varían de $2.38 \mathrm{~mm}$ de longitud mínima a $8.58 \mathrm{~mm}$ de longitud máxima en ejemplares completos.

La geometría de la acumulación es en capas y la estructura interna es simple, pues la concentración esqueletal es homogénea internamente.

Grados tafonómicos: Los gasterópodos presentan grados tafonómicos que varían de muy altos (A) a altos (B) (Cuadro 1).

El grado tafonómico "A" caracteriza a este estrato debido a la escasa corrasión y la orientación aleatoria (alineación paralela de menos del $10 \%$ ) de los ejemplares, así como a la ausencia de gradación del sedimento. El grado tafonómico "B" corresponde al porcentaje de

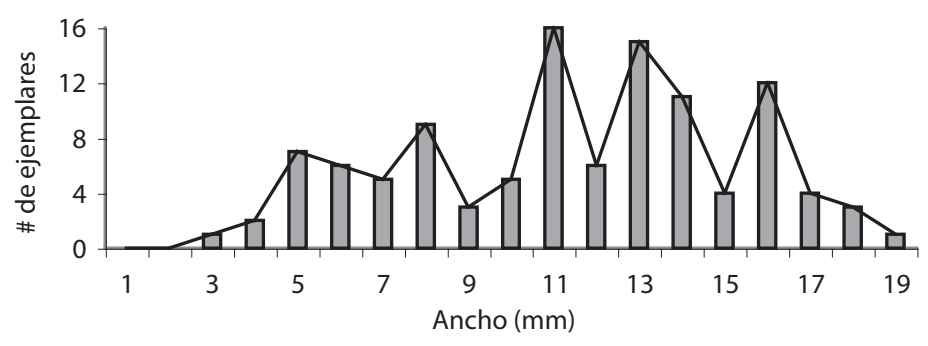

Fig. 3. Distribución de frecuencia de la anchura de la muestra del gasterópodo cf. Donaldina robusta.

Fig. 3. Size frequency distribution from the cf. Donaldina robusta gastropod assemblage. 


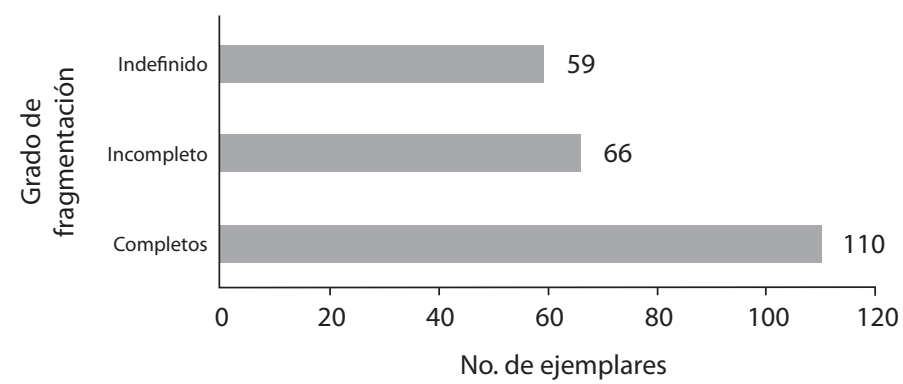

Fig. 4. Porcentaje de fragmentación de las conchas del gasterópodo cf. Donaldina robusta.

Fig. 4. Fragmentation percentages of the shells of cf. Donaldina robusta gastropod.

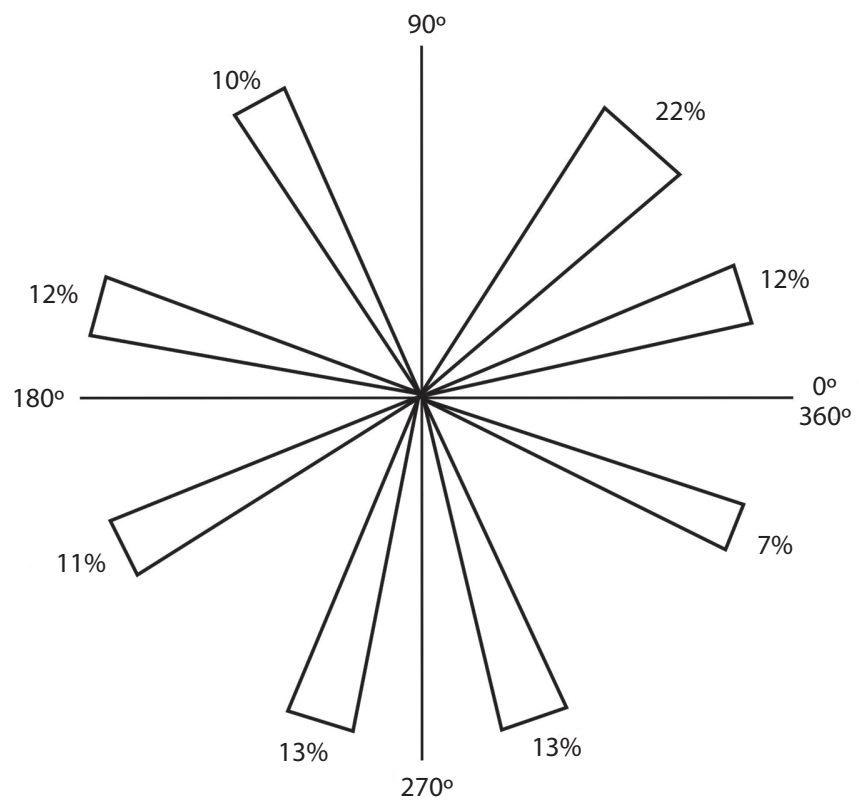

Fig. 5. Ángulo de orientación de 176 gasterópodos.

Fig. 5. Orientation patterns of 176 gastropod shells.

fragmentación de los ejemplares (28\%), el porcentaje de matriz (roca tipo "wackestone") y la clasificación de la matriz y los aloquímicos de la muestra. En el tafograma ternario (Fig. 6) se comparan las características consideradas para este análisis.

\section{DISCUSIÓN}

De acuerdo al histograma y curva de frecuencia se considera que el yacimiento es de tipo monotípico y monoespecífico pues presenta el comportamiento de una comunidad típica, con pocos ejemplares de tallas pequeñas y grandes y la mayoría distribuidos en tallas intermedias.

Los grados tafonómicos son indicadores de la cantidad de retrabajo en los organismos. La escasa corrasión en los ejemplares indica un tiempo mínimo de exposición de los restos en la interfase agua-sedimento y por 
CUADRO 1

Grados tafonómicos de la acumulación de cf. Donaldina robusta en la Formación La Joya

TABLE 1

Taphonomic grades from the assemblages of $c f$. Donaldina robusta in the La Joya Formation

Características tafonómicas

Grados tafonómicos

$\%$ Fragmentación

B $(28 \%)$

$\%$ Corrasión

A $(<10)$

$\%$ Orientación

A $(<10)$

Características Sedimentológicas

$\%$ Matriz

Grados tafonómicos

Clasificación

B (75-90)

Gradación

B (1-0.5)

$\mathrm{A}(<10)$

\section{$\triangle$ Fragmentación \\ $\triangle$ Corrasión \\ Orientación \\ $\diamond$ Matriz \\ \& Clasificación \\ $\square$ Gradación}

nón

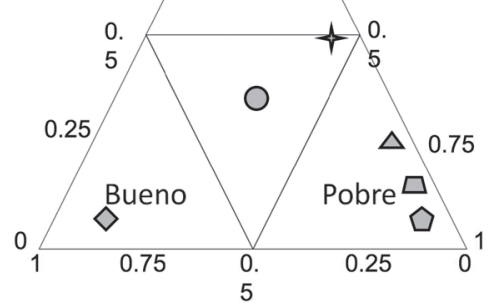

Fig. 6. Tafograma ternario donde se comparan las características tafonómicas del conjunto de gasterópodos.

Fig. 6. Ternary taphogram comparing the taphonomic features of the gastropod assemblage.

tanto un enterramiento no muy lento (Brett \& Baird 1986), también es indicadora de poco disturbio posmortem pues el transporte de los restos ocasiona abrasión física (Olsewski \& West 1997). La orientación aleatoria puede ser indicadora de ambientes de baja energía (Salazar et al. 1982), de sustratos bioturbados (Toots 1965) o de enterramiento en un flujo de granos (Wider 1968), en el conjunto estudiado no hay evidencia de flujo de granos, por lo cual la orientación aleatoria y sin imbricación de los ejemplares indica la ausencia de una corriente en un ambiente de baja energía.

El grado de fragmentación de los organismos es bajo (28\%) además de que la fractura se dio en una zona preferencial, el ápice que es el área más frágil en las conchas, lo cual puede indicar que tuvieron retrabajos episódicos y estuvieron expuestos a periodos cortos de exhumación (Brett \& Baird 1986), sin embargo hay que considerar que el ápice también se desgasta en forma natural durante la vida del organismo, sin necesidad de retrabajo post-mortem.

La mala clasificación de los aloquímicos muestra que se trata de un conjunto sin disturbio, pues la presencia de conchas pequeñas (juveniles) junto a conchas grandes (adultos) sugiere una población paraautóctona que no ha sido sometida a procesos destructivos (Olsewski \& West 1997).

De acuerdo al grado tafonómico intermedio A/B de la acumulación de gasterópodos en la Formación La Joya se interpreta que los organismos tuvieron mínimo disturbio postmortem relacionado a poco retrabajo y escasa transportación, y que el depósito se dio en una zona de baja energía. El tiempo de resolución temporal es escaso, se trata por tanto de un depósito de corto tiempo, indicado por la gran cantidad de matriz y la ausencia de gradación (Brandt 1989), esta inferencia es apoyada porque la distribución que muestran los organismos dentro del estrato es muy dispersa y no se encuentran en capas lo que indica una acumulación de tipo natural sin influencia de corrientes o tormentas, y lateralmente el estrato es consistente con esta característica. Sin embargo, hay que considerar que se ha demostrado a través de estudios actuotafonómicos en moluscos, que puede haber una diferencia de miles de años entre organismos que se encuentran uno junto a otro en un mismo estrato (Flessa \& Kowalewski 1994, Kowalewski et al. 1998). De acuerdo a la clasificación biostratinómica de las concentraciones esqueletales propuesta por Kidwell et al. (1986) estos atributos tafonómicos caracterizan a un conjunto de tipo sedimentológico que corresponde a una acumulación de partes 
duras durante un periodo de sedimentación neta baja, que origina la acumulación de especímenes autóctonos/paraautóctonos, que han sido retrabajados en cierto grado pero sin ser transportados fuera de su hábitat original.

Las concentraciones de tipo sedimentológico se consideran comunes en zonas de plataforma abierta por encima de la base de la ola (Kidwell et al. 1986).

El conjunto fósil de la Formación La Joya es típico de la biota del Pensilvánico que predominó en la mayoría de plataformas carbonatadas someras, muy común en las regiones continentales medias del Paleozoico Tardío (Gómez et al. 2008).

De acuerdo al grado tafonómico intermedio de muy alto a alto $(\mathrm{A} / \mathrm{B})$ que se presenta en este afloramiento, se interpreta que el material tuvo poco retrabajo y escasa transportación, debido a episodios de exhumación. Las características biostratinómicas (orientación aleatoria, bajo porcentaje de fragmentación y corrasión) y sedimentológicas (mala clasificación y alto porcentaje de matriz) son indicadores de una rápida depositación en condiciones de baja energía.

La acumulación del gasterópodo cf. Donaldina robusta en la Formación La Joya en Sierra Agua Verde corresponde a un depósito de tipo sedimentológico conformado por organismos autóctonos/paraautóctonos.

\section{AGRADECIMIENTOS}

Al Posgrado en Ciencias Biológicas, UNAM; al CONACYT y la DGEP, UNAM por el financiamiento de Catalina Gómez. Al CONACYT (Proyecto 49088), UNAMPAPIIT (Proyecto IN118209) y ECOSANUIES-CONACyT (Proyecto M06-U01) por el financiamiento del presente trabajo. A Emilio Almazán por la asesoría durante el trabajo de campo. A Antonieta Carrasco y Juan Guillermo Casillas por la edición de imágenes.

\section{RESUMEN}

Los gasterópodos son un componente importante en la mayoría de los registros fósiles, sin embargo, las investigaciones se han centrado principalmente en la caracterización de las tafofacies y firmas tafonómicas en ambientes particulares. Se presentan los datos tafonómicos cualitativos y cuantitativos para el ensamblaje del gasterópodo cf. Donaldina robusta de la Formación La Joya de la Sierra Agua Verde, Sonora, (NW) México. Se analizaron 176 conchas. Una buena preservación recibió un grado tafonómico alto (A) y una preservación pobre D. Las conchas estaban completas en el $72 \%$ de los casos (grado tafonómico B). Menos del $10 \%$ estaban corroídas y se encontraban paralelas a la capa (grado A). Esta roca según la petrografía es clasificada como "wackestone", de acuerdo a los sedimentos se encuentra en una categoría intermedia (grado B) y baja (grado A). Los ensambles fosilíferos tienen grados tanto A como B. Las características biostratinómicas del ensamblaje esquelético tienen concentraciones de sedimentos de los elementos autóctonos-parautóctonos típicas del sitio de la acumulación. Durante un corto período de acumulación, en un ambiente de baja energía, hubo reelaboración y transporte mínimo de la producción local.

Palabras clave: biostratinomía, grados tafonómicos, paleoecología, gasterópodos, Pensilvánico, Sonora.

\section{REFERENCIAS}

Almazán, E., D. Vachard, B.E. Buitrón, C. Mendoza \& C. Gómez-Espinosa. 2007. The late Atokan (Moscovian-Pennsylvanian) chaetetid accumulations of Sierra Agua Verde, Sonora (NW Mexico): composition, facies and paleoenvironmental signals, p. 189-200. In J.J. Alvaro, M. Aretz, F. Boulvain, A. Munnecke, D. Vachard \& E. Vennin (eds.). Paleozoic Reefs and Bioaccumulations: Climatic and Evolutionary Controls. Geol. Soc. London. Londres, Inglaterra.

Behrensmeyer, A.K. 1984. Taphonomy and the fossil record. Am. Sci. 72: 558-565.

Brandt, D.S. 1989. Taphonomic grades as a classification for fossiliferous assemblages and implications for paleoecology. Palaios 4: 303-309.

Brett, C.E. \& G.C. Baird. 1986. Comparative taphonomy: a key to paleoenvironmental interpretation based on fossil preservation. Palaios 1: 27-227.

Buitrón, B.E., C. Gómez, E. Almazán \& D. Vachard. 2007. A late Atokan regional encrinite (early late 
Moscovian, Middle Pennsylvanian) in the Sierra Agua Verde, Sonora State, NW México, p. 201-209. In J. J. Alvaro, M. Aretz, F. Boulvain, A. Munnecke, D. Vachard \& E. Vennin (eds.). Paleozoic Reefs and Bioaccumulations: Climatic and Evolutionary Controls. Geol. Soc. London. Londres, Inglaterra.

Carlson, E.H. 1994. Paleoshoreline patterns in the transgressive-regressive sequences of Pennsylvanian rocks in the northern Appalachian Basin, U.S.A. Sed. Geol. 93: 209-222.

Davies, D.J., G.M. Staff, W.R. Callender \& E.N. Powell, 1990. Description of a quantitative approach to taphonomy and taphofacies analysis: All dead things are not created equal, p. 328-350. In W. Miller (ed.). Paleocommunity temporal dynamics: The long-term development of multispecies assemblies. Pal. Soc., Universidad de Tennessee, Knoxville, EEUU.

Dunham, R.J. 1962. Classification of carbonate rocks according to depositional texture, 108-1212. In W. E. Ham (ed.). Classification of Carbonate Rocks. AAPG Memoir 1.

Flessa, K.W. \& M. Kowalewski. 1994. Shell survival and time averaging in near shore and shelf environments: estimates from the radiocarbon literature. Lethaia 27: 153-163.

Gómez, C., D. Vachard, B. Buitrón, E. Almazán \& C. Mendoza. 2008. Pennsylvanian fusulinids and calcareous algae from Sonora (northwestern Mexico), and their biostratigraphic and palaeobiogeographic implications. Palevol 7: 259-268.

Heckel, P.H. 1983. Diagenetic model for carbonate rocks in Midcontinent Upper Pennsylvannian cyclothems. J. Sed. Pet. 53: 733-759.

Heckel, P.H. 1986. Sea-level curve for Pennsylvanian eustatic marine transgressive-regressive depositional cycles along midcontinent outcrop belt, North America. Geology 14: 330-334.

Kidwell, S.M., F.T. Fürsich \& T. Aigner. 1986. Conceptual framework for the analysis and classification of fossil concentrations. Palaios 1: 228-238.
Kidwell, S.M., T.A. Rothfus \& M.R. Best. 2001. Sensitivity of taphonomic signatures to sample size, sieve size, damage scoring system, and target taxa. Palaios 16: $26-52$.

Kowalewski, M., G.A. Goodfriends \& K.W. Flessa. 1998. High resolution estimates of temporal mixing within shell beds: the evils and virtues of time averaging. Paleobiology 24: 287-304.

Ochoa, A. \& P. Sosa. 1993. Geología y estratigrafía de la Sierra Agua Verde, con énfasis en el Paleozoico. Tesis de Licenciatura. Universidad de Sonora, Sonora, México.

Olszewski, T. \& R.R. West. 1997. Influence of transportation and time-averaging in fossil assemblages from the Pennsylvanian of Oklahoma. Lethaia 30: 315-329.

Salazar, A., R.W. Frey \& J.D. Howard. 1982. Concavity orientation of bivalve shells in estuarine and nearshore shelf sediments, Georgia. J. Sed. Pet. 52: 565-586.

Speyer, S.E. \& C.E. Brett. 1988. Taphofacies models for epeiric sea environments: Middle Paleozoic examples. Paleogeo. Paleoclim. Paleoecol. 63: 225-262.

Toots, H. 1965. Random orientation of fossils and its significance. Rocky Mountain Geol. 4: 59-62.

Walker S.E. \& J.T. Carlton. 1995. Taphonomic losses become taphonomic gains: an experimental approach using the rocky shore gastropod, Tegula funebralis. Paleogeo. Paleoclim. Paleoecol. 114: 197-217.

Wider, C.G. 1968. Carbonate diagenesis by burrowing organisms. 23rd International Geological Congress Report, Proceeding of Simposium Praga, Checoslovaquia: $133-140 \mathrm{p}$.

Wilde, G.L. 1990. Practical fusulinid zonation: the species concept, with Permian Basin. West Texas Geol. Soc. Bull. 29: 5-34.

Zuschin, M., M. Stachowitsch \& R. Stanton, Jr. 2003. Patterns and processes of shell fragmentation in modern and ancient marine environments. Earth-Science Rev. 63: $33-82$. 
APENDICE 1

Datos de medidas de las conchas y características biostratinómicas analizadas del gasterópodo cf. Donaldina robusta

APENDIX 1

Data for size and biostratinomic features from $c f$. Donaldina robusta gastropod

\begin{tabular}{|c|c|c|c|c|c|}
\hline$\#$ & Alto (mm) & Ancho (mm) & Orientación $\left({ }^{\circ}\right)$ & Fragmentación* & Corrasión** \\
\hline 1 & 7.15 & 2.99 & 12 & $\mathrm{C}$ & $\mathrm{B}$ \\
\hline 2 & 7.67 & 3.64 & 223 & $\mathrm{C}$ & $\mathrm{B}$ \\
\hline 3 & 7.93 & 3.25 & 60 & $\mathrm{C}$ & $\mathrm{B}$ \\
\hline 4 & 7.8 & 2.6 & 43 & $\mathrm{C}$ & $\mathrm{B}$ \\
\hline 5 & 6.89 & 2.73 & 163 & $\mathrm{C}$ & $\mathrm{B}$ \\
\hline 6 & 5.2 & 2.47 & 305 & $\mathrm{C}$ & $\mathrm{B}$ \\
\hline 7 & 6.76 & 2.6 & 197 & $\mathrm{C}$ & B \\
\hline 8 & 6.24 & 3.25 & 193 & $\mathrm{C}$ & B \\
\hline 9 & 7.41 & 3.25 & 217 & $\mathrm{C}$ & $\mathrm{B}$ \\
\hline 10 & 6.63 & 2.73 & 13 & $\mathrm{C}$ & $\mathrm{B}$ \\
\hline 11 & 8.19 & 2.73 & 11 & $\mathrm{C}$ & $\mathrm{B}$ \\
\hline 12 & 5.46 & 2.21 & 230 & $\mathrm{C}$ & B \\
\hline 13 & 6.5 & 2.73 & 313 & $\mathrm{C}$ & B \\
\hline 14 & 4.94 & 2.86 & 350 & $\mathrm{C}$ & $\mathrm{B}$ \\
\hline 15 & 8.00 & 3.8 & 272 & $\mathrm{C}$ & $\mathrm{B}$ \\
\hline 16 & 8.58 & 3.77 & 69 & $\mathrm{C}$ & M \\
\hline 17 & 7.8 & 3.25 & 231 & $\mathrm{C}$ & B \\
\hline 28 & 7.8 & 3.9 & 317 & $\mathrm{C}$ & B \\
\hline 19 & 8.45 & 4.03 & 280 & $\mathrm{C}$ & B \\
\hline 20 & 7.93 & 2.99 & 72 & $\mathrm{C}$ & B \\
\hline 21 & 7.02 & 3.9 & 66 & $\mathrm{C}$ & B \\
\hline 22 & 7.54 & 3.25 & 110 & $\mathrm{C}$ & B \\
\hline 23 & 5.85 & 2.86 & 338 & $\mathrm{C}$ & B \\
\hline 24 & 6.5 & 3.38 & 62 & $\mathrm{C}$ & A \\
\hline 25 & 6.37 & 2.73 & 350 & $\mathrm{C}$ & B \\
\hline 26 & 6.76 & 2.99 & 152 & $\mathrm{C}$ & B \\
\hline 27 & 5.2 & 2.86 & 35 & $\mathrm{C}$ & B \\
\hline 28 & 6.76 & 3.38 & 304 & $\mathrm{C}$ & B \\
\hline 29 & 7.28 & 3.25 & 285 & $\mathrm{C}$ & B \\
\hline 30 & 5.59 & 2.86 & 135 & $\mathrm{C}$ & M \\
\hline 31 & 8.45 & 2.47 & 115 & $\mathrm{C}$ & B \\
\hline 32 & 7.8 & 3.9 & 219 & $\mathrm{C}$ & B \\
\hline 33 & 8.97 & 3.9 & 69 & $\mathrm{C}$ & B \\
\hline 34 & 8.45 & 3.9 & 250 & $\mathrm{C}$ & B \\
\hline 35 & 7.02 & 3.64 & 94 & $\mathrm{C}$ & B \\
\hline 36 & 7.54 & 4.16 & 311 & $\mathrm{C}$ & B \\
\hline 37 & 6.37 & 2.73 & 157 & $\mathrm{C}$ & B \\
\hline 38 & 8.06 & 4.03 & 86 & $\mathrm{C}$ & B \\
\hline 39 & 8.58 & 4.16 & 63 & $\mathrm{C}$ & B \\
\hline 40 & 5.2 & 2.47 & 338 & $\mathrm{C}$ & B \\
\hline 41 & 5.46 & 4.95 & 84 & $\mathrm{C}$ & M \\
\hline 42 & 6.37 & 2.99 & 94 & $\mathrm{C}$ & B \\
\hline 43 & 3.64 & 2.34 & 307 & $\mathrm{C}$ & B \\
\hline 44 & 5.2 & 2.73 & 88 & $\mathrm{C}$ & B \\
\hline 45 & 6.37 & 3.51 & 332 & $\mathrm{C}$ & A \\
\hline 46 & 7.15 & 3.38 & 288 & $\mathrm{C}$ & B \\
\hline
\end{tabular}


APENDICE 1 (Continuación)

Datos de medidas de las conchas y características biostratinómicas analizadas del gasterópodo cf. Donaldina robusta

APENDIX 1 (Continued)

Data for size and biostratinomic features from $c f$. Donaldina robusta gastropod

\begin{tabular}{|c|c|c|c|c|c|}
\hline \# & Alto (mm) & Ancho (mm) & Orientación $\left({ }^{\circ}\right)$ & Fragmentación* & Corrasión** \\
\hline 47 & 6.76 & 2.6 & 129 & $\mathrm{C}$ & B \\
\hline 48 & 7.8 & 2.86 & 100 & $\mathrm{C}$ & B \\
\hline 49 & 3.78 & 2.36 & 54 & $\mathrm{C}$ & B \\
\hline 50 & 7.8 & 3.9 & 164 & $\mathrm{C}$ & M \\
\hline 51 & 4.94 & 2.47 & 54 & $\mathrm{C}$ & B \\
\hline 52 & 5.2 & 2.47 & 149 & $\mathrm{C}$ & $\mathrm{B}$ \\
\hline 53 & 5.2 & 3.25 & 340 & $\mathrm{C}$ & B \\
\hline 54 & 5.07 & 3.25 & 148 & $\mathrm{C}$ & B \\
\hline 55 & 5.85 & 2.99 & 320 & $\mathrm{C}$ & B \\
\hline 56 & 5.07 & 2.86 & 159 & $\mathrm{C}$ & B \\
\hline 57 & 6.5 & 2.47 & 48 & $\mathrm{C}$ & $\mathrm{B}$ \\
\hline 58 & 6.76 & 3.38 & 313 & $\mathrm{C}$ & B \\
\hline 59 & 6.5 & 2.86 & 252 & $\mathrm{C}$ & B \\
\hline 60 & 5.59 & 2.6 & 68 & $\mathrm{C}$ & B \\
\hline 61 & 7.8 & 4.55 & 6 & $\mathrm{C}$ & B \\
\hline 62 & 5.2 & 2.47 & 7 & $\mathrm{C}$ & B \\
\hline 63 & 9.1 & 4.16 & 204 & $\mathrm{C}$ & B \\
\hline 64 & 5.2 & 2.47 & 67 & $\mathrm{C}$ & M \\
\hline 65 & 3.30 & 1.98 & 232 & $\mathrm{C}$ & B \\
\hline 66 & 2.36 & 1.89 & 281 & $\mathrm{C}$ & B \\
\hline 67 & 2.93 & 1.70 & 256 & $\mathrm{C}$ & B \\
\hline 68 & 4.25 & 2.60 & 143 & $\mathrm{C}$ & B \\
\hline 69 & 3.59 & 1.51 & 66 & $\mathrm{C}$ & B \\
\hline 70 & 1.41 & 1.27 & 128 & $\mathrm{C}$ & B \\
\hline 71 & 2.36 & 1.21 & 274 & $\mathrm{C}$ & B \\
\hline 72 & 2.83 & 1.51 & 69 & $\mathrm{C}$ & B \\
\hline 73 & 3.12 & 1.67 & 337 & $\mathrm{C}$ & B \\
\hline 74 & 6.14 & 2.83 & 310 & $\mathrm{C}$ & B \\
\hline 75 & 4.25 & 2.36 & 207 & $\mathrm{C}$ & B \\
\hline 76 & 5.67 & 2.83 & 228 & $\mathrm{C}$ & B \\
\hline 77 & 6.14 & 2.84 & 187 & $\mathrm{C}$ & B \\
\hline 78 & 3.30 & 1.70 & 54 & $\mathrm{C}$ & B \\
\hline 79 & 5.85 & 1.98 & 119 & $\mathrm{C}$ & B \\
\hline 80 & 6.37 & 2.47 & 162 & $\mathrm{C}$ & B \\
\hline 81 & 5.2 & 2.34 & 62 & $\mathrm{C}$ & B \\
\hline 82 & 6.76 & 2.21 & 225 & $\mathrm{C}$ & B \\
\hline 83 & 6.37 & 1.56 & 234 & $\mathrm{C}$ & B \\
\hline 84 & 6.76 & 2.73 & 8 & $\mathrm{C}$ & B \\
\hline 85 & 6.5 & 2.73 & 44 & $\mathrm{C}$ & $\mathrm{M}$ \\
\hline 86 & 5.07 & 2.73 & 300 & $\mathrm{C}$ & B \\
\hline 87 & 4.25 & 2.73 & 162 & $\mathrm{C}$ & B \\
\hline 88 & 5.2 & 2.34 & 163 & $\mathrm{C}$ & B \\
\hline 89 & 5.2 & 2.73 & 290 & $\mathrm{C}$ & B \\
\hline 90 & 5.85 & 3.50 & 115 & $\mathrm{C}$ & B \\
\hline 91 & 3.9 & 1.95 & 77 & $\mathrm{C}$ & B \\
\hline
\end{tabular}


APENDICE 1 (Continuación)

Datos de medidas de las conchas y características biostratinómicas analizadas del gasterópodo cf. Donaldina robusta

APENDIX 1 (Continued)

Data for size and biostratinomic features from cf. Donaldina robusta gastropod

\begin{tabular}{|c|c|c|c|c|c|}
\hline \# & Alto (mm) & Ancho (mm) & Orientación $\left({ }^{\circ}\right)$ & Fragmentación* & Corrasión** \\
\hline 92 & 3.51 & 1.56 & 294 & $\mathrm{C}$ & $\mathrm{B}$ \\
\hline 93 & 3.9 & 2.47 & 3 & $\mathrm{C}$ & $\mathrm{B}$ \\
\hline 94 & 5.85 & 2.73 & 177 & $\mathrm{C}$ & B \\
\hline 95 & 2.47 & 2.73 & 39 & $\mathrm{C}$ & B \\
\hline 96 & 3.9 & 1.95 & 251 & $\mathrm{C}$ & B \\
\hline 97 & 3.9 & 2.21 & 27 & $\mathrm{C}$ & B \\
\hline 98 & 1.69 & 1.56 & 183 & $\mathrm{C}$ & B \\
\hline 99 & 4.03 & 1.69 & 108 & $\mathrm{C}$ & B \\
\hline 100 & 6.5 & 2.99 & 252 & $\mathrm{C}$ & B \\
\hline 101 & 5.59 & 2.6 & 259 & $\mathrm{C}$ & B \\
\hline 102 & 2.73 & 2.21 & 144 & $\mathrm{C}$ & B \\
\hline 103 & 2.60 & 1.95 & 70 & $\mathrm{C}$ & B \\
\hline 104 & 3.9 & 2.21 & 237 & $\mathrm{C}$ & B \\
\hline 105 & 2.73 & 1.82 & 12 & $\mathrm{C}$ & B \\
\hline 106 & 2.8 & 1.62 & 314 & $\mathrm{C}$ & $\mathrm{B}$ \\
\hline 107 & 2.4 & 1.56 & 311 & $\mathrm{C}$ & B \\
\hline 108 & 2.4 & 1.62 & 239 & $\mathrm{C}$ & B \\
\hline 109 & 2.00 & 1.62 & 157 & $\mathrm{C}$ & $\mathrm{M}$ \\
\hline 110 & 2.4 & 1.62 & 318 & $\mathrm{C}$ & B \\
\hline 111 & & & 265 & I & B \\
\hline 112 & & & 70 & I & B \\
\hline 113 & & & 82 & I & B \\
\hline 114 & & & 262 & I & B \\
\hline 115 & & & 202 & I & B \\
\hline 116 & & & 269 & I & B \\
\hline 117 & & & 29 & I & M \\
\hline 118 & & & 153 & I & B \\
\hline 119 & & & 59 & I & B \\
\hline 120 & & & 291 & I & B \\
\hline 121 & & & 171 & I & B \\
\hline 122 & & & 260 & I & B \\
\hline 123 & & & 218 & I & B \\
\hline 124 & & & 82 & I & B \\
\hline 125 & & & 144 & I & B \\
\hline 126 & & & 79 & I & M \\
\hline 127 & & & 328 & I & B \\
\hline 128 & & & 83 & I & B \\
\hline 129 & & & 156 & I & B \\
\hline 130 & & & 28 & I & B \\
\hline 131 & & & 310 & I & B \\
\hline 132 & & & 206 & I & B \\
\hline 133 & & & 127 & I & B \\
\hline 134 & & & 196 & I & B \\
\hline 135 & & & 82 & I & B \\
\hline 136 & & & 116 & I & B \\
\hline
\end{tabular}


APENDICE 1 (Continuación)

Datos de medidas de las conchas y características biostratinómicas analizadas del gasterópodo cf. Donaldina robusta

APENDIX 1 (Continued)

Data for size and biostratinomic features from $c f$. Donaldina robusta gastropod

\begin{tabular}{|c|c|c|c|c|}
\hline \# & Alto $(\mathrm{mm}) \quad$ Ancho $(\mathrm{mm})$ & Orientación $\left({ }^{\circ}\right)$ & Fragmentación* & Corrasión** \\
\hline 137 & & 36 & I & M \\
\hline 138 & & 238 & I & $\mathrm{B}$ \\
\hline 139 & & 162 & I & B \\
\hline 140 & & 22 & I & B \\
\hline 141 & & 223 & I & B \\
\hline 142 & & 114 & I & B \\
\hline 143 & & 135 & I & B \\
\hline 144 & & 116 & I & B \\
\hline 145 & & 155 & I & B \\
\hline 146 & & 96 & I & B \\
\hline 147 & & 63 & I & $\mathrm{B}$ \\
\hline 148 & & 146 & I & $\mathrm{B}$ \\
\hline 149 & & 196 & I & B \\
\hline 150 & & 261 & I & $\mathrm{B}$ \\
\hline 151 & & 147 & I & $\mathrm{M}$ \\
\hline 152 & & 232 & I & B \\
\hline 153 & & 71 & I & B \\
\hline 154 & & 21 & I & B \\
\hline 155 & & 80 & I & B \\
\hline 156 & & 233 & I & B \\
\hline 157 & & 270 & I & B \\
\hline 158 & & 58 & I & B \\
\hline 159 & & 54 & I & B \\
\hline 160 & & 67 & I & B \\
\hline 161 & & 51 & I & B \\
\hline 162 & & 219 & I & B \\
\hline 163 & & 33 & I & $\mathrm{M}$ \\
\hline 164 & & 215 & I & B \\
\hline 165 & & 68 & I & B \\
\hline 166 & & 198 & I & B \\
\hline 167 & & 57 & I & B \\
\hline 168 & & 300 & I & B \\
\hline 169 & & 230 & I & B \\
\hline 170 & & 202 & I & B \\
\hline 171 & & 44 & I & B \\
\hline 172 & & 63 & I & B \\
\hline 173 & & 8 & I & B \\
\hline 174 & & 328 & I & B \\
\hline 175 & & 311 & I & B \\
\hline 176 & & 9 & I & $\mathrm{M}$ \\
\hline
\end{tabular}

